\title{
Candida biofilm - A medical havoc: Prospective study among the patients in tertiary health care centre in North India
}

\author{
Vikramjeet Singh $^{1}$, Kriti Maurya ${ }^{2}$, Manodeep Sen $^{3}$, Anupam Das ${ }^{4, *}$, Jyotsna Agarwal $^{5}$ \\ ${ }^{1}$ Senior Resident, ${ }^{2}$ Post Graduate Resident, ${ }^{3}$ Additional Professor, ${ }^{4}$ Associate Professor, ${ }^{5}$ Professor \& HOD, \\ Dept. of Microbiology, Dr. Ram Manohar Lohia Institute of Medical Sciences, Lucknow, Uttar Pradesh, India
}

*Corresponding Author:

Email: dasdranupam@gmail.com

\begin{abstract}
Objective of this study is to know the incidence of biofilm formation by isolates of Candida spp among the patients attending Tertiary health care Superspeciality centre in Lucknow.

Material and Methods: A prospective study conducted among patients attending Out-patient department and admitted to Inpatient department, and Intensive care unit during the period between January $1^{\text {st }}$ and December $31^{\text {st }} 2017$. Different samples like endotracheal secretion, urine, blood and pus were collected under sterile conditions and standard fungal tests were performed for identification and appropriate statistical methods were employed to look for association between biofilm and Candida species.

Results: Out of the 216 samples included in the study from various departments, 80 samples showed evidence of Candida infections yielding an infection rate of 37\%. Among the 99 isolates (32 Candida albicans and 67 Non albicans Candida) subjected for biofilm production, 52 (52.5\%) were positive.

Conclusion: Non albicans Candida infection has drastically increased in healthcare centres. Biofilms have significant role in the perpetuation of these infections and antifungal resistance primarily with respect to their ability to adhere to various medical devices. Regular monitoring on the sensitivity and biofilm formation will be helpful in optimising therapy and outcome.
\end{abstract}

Keywords: Candida Spp, Biofilms, Microtitre Plate Method.

\section{Introduction}

Candida species are frequently encountered in the normal microbiota of humans, however, in cases of immunocompromised host these fungal pathogens facilitates their invasion over medical devices and host surfaces. Non albicans Candida species are now emerging as major agents of hospital acquired infections due to dramatic rise in organ transplant patients, cancer patients and HIV patients. Although C. albicans is the predominant etiologic agent of candidiasis, other Candida species that tend to be less susceptible to the commonly used antifungal drugs such as C. tropicalis, C.krusei, C. glabrata, C. lusitaniae, etc. have emerged as substantial opportunistic pathogens. Non albicans Candida shares many virulence factors with C. albicans such as biofilm production and phenotypic switching. ${ }^{1}$

Biofilms are specific and organized communities of cells under the control of signalling molecules, rather than random accumulations of cells resulting from cell division. Biofilm produced on medical devices can negatively impact the host immune defences by causing the failure of the device, enhanced resistance against most antifungal agents and by serving as a reservoir or source for future continuing infections. ${ }^{2}$ In the expanding population of immunocompromised persons and inappropriate use of antifungal treatment, there is a drastic increase in antifungal drug resistance leading to morbidity and increase mortality of patients. ${ }^{3,4}$

The rationale of this study was to determine the incidence of biofilm formation by various Candida species isolated from clinical specimens among patients attending tertiary health care centre.

\section{Material and Methods}

This was a hospital based prospective study conducted in the Department of Microbiology, of a tertiary care super-Specialty hospital. A total of 216 clinical samples like blood, urine, pus and endotracheal secretions from IPD, OPD and ICU were included in the study. All the suspected Candida isolates were confirmed using the tests mentioned and only those isolates with confirmed identity were included for the further biofilm analysis.

The Candida isolates were inoculated on SCCA, SDA and incubated at $25^{\circ} \mathrm{C}$ and $37^{\circ} \mathrm{C}$ for 7 days respectively. Colony morphology was observed with difference in colony, size and margin for different species on the SDA, Hicrome agar (Fig. 1) and CMA. ${ }^{5}$ Isolated Candida spp. were identified by colony morphology, Gram staining, Germ tube test and appearance of Chlamydospore on cornmeal agar (Fig. 2).

\section{Biofilm formation}

\section{A. Tube method}

A qualitative assessment of biofilm formation was determined by following method ${ }^{9}$. BHIB $(10 \mathrm{ml})$ was inoculated with loopful of Candida from overnight culture plates and incubated for $24 \mathrm{~h}$ at $37^{\circ} \mathrm{C}$. The tubes were decanted and washed with phosphate buffered saline (PBS, pH 7.3) and dried. Dried tubes were 
stained with crystal violet $(0.1 \%)$. Excess stain was removed and washed with distilled water. Tubes were dried in inverted position and observed for biofilm formation. Biofilm formation was considered positive when presence of visible film appeared or lined on the wall and bottom of the tube. Ring formation at the liquid interface was not considered as an indication of biofilm production.

\section{B. Micro-titre Plate Method}

The microtitre plate assay is the most widely used method and considered as standard test for the detection of biofilm formation. ${ }^{9}$ Individual wells of sterile, polystyrene, 96 well-flat bottom microtitre plates were filled with $100 \mu \mathrm{l}$ aliquots of the cell suspension and only broth served as control to check sterility and nonspecific binding of media. The microtitre plates then incubated for $72 \mathrm{~h}$ at $37^{\circ} \mathrm{C}$. After incubation content of each well were gently removed by tapping the plates. The wells were washed four times with $200 \mu \mathrm{l}$ of PBS ( $\mathrm{pH}$ 7.2) to remove free-floating 'planktonic' organism. Biofilms formed by adherent 'sessile' organisms in plate which were stained with crystal violet $(0.1 \% \mathrm{w} / \mathrm{v})$. Excess stain was rinsed off by thorough washing with distilled water and plates were kept for drying. Adherent Candida cells usually formed a biofilm at the bottom of the wells. Optical density (OD) of stained adherent Candida biofilm will be determined with a micro ELISA auto reader. (Fig. 3)

\section{Results}

During this study period, total of 99 isolates of Candida species were obtained from 80 samples out of 216 different clinical specimens of patients admitted in various department. Of these, $143(66 \%)$ from urine of patients, $53(25 \%)$ from blood, $11(5 \%)$ were from Endotracheal secretions and $9(4 \%)$ from pus. Urine and Blood isolates had more predilection for biofilm formation.

The study indicated slight preponderance of females $(53 \%)$ in Candida infection than males $(47 \%)$. The most common age group of patients which was affected by Candida infections in ICU were 40-60 years (36.8\%) followed by elderly age group more than 60 years $(26.5 \%)$.However, demographic difference in age and sex distribution was not statistically significant $(p>0.05)$.

The distribution among 99 Candida species isolated were C. albicans [32 (33.3\%)], C. glabrata [22 (21.8\%)], C.dubliniensis [13 (12.8\%)], C.guilliermondii [12 (11.78\%], C. krusei [9 (8.9\%)], C. tropicalis [7 $(7 \%)]$, C. kefyr $[3(2.97 \%)]$ and C. parapsilosis [1 (1\%)].

Among the 99 isolates subjected for biofilm production, $52(52.5 \%)$ were positive. Biofilm was strongly produced in 18 strains most commonly by C.tropicalis, C.albicans and C.parapsilosis, however, 34 strains identified as weak to moderate biofilm producers like C.glabrata, C.lusitaniae and C. krusei. (Fig. 4)

Among the total positive samples that were subjected to tube method and microtitre plate method for biofilm formation, $18.18 \%$ in Tube method and $14.14 \%$ in Microtitre plate were found to be strong biofilm producers. Total biofilm producers detected by tube method were $45 \%$ where as in microtitre plate method it was increased to $53 \% \quad(\mathrm{p}<0.05)$. In the standard MTP assay, from the total number of 99 isolates tested for biofilm formation, strong biofilm producers were $14(14.14 \%), 16$ (16.16\%) were moderate and $13(13.13 \%)$ isolates were considered as weak biofilm producers. Among total isolates, 56 isolates were not able to produce biofilm on microtitre plate. The fourteen strong biofilm producers showed significant optical density values of more than 0.320 in microtitre plate assay. Most commonly strong biofilm producers were Candida tropicalis and Candida albicans. (Table 1)

In the present study using tube method for biofilm detection, a total of $18(23.33 \%)$ isolates were picked up as strong and $9(70 \%)$ were moderate and 9 isolates were weak biofilm producers. However, it was difficult to discriminate between moderate and weak biofilm producing isolates on tube method. By adopting tube method 63 isolates were considered as non biofilm producing isolates.

As few cases were from the ICU, the presence of various risk factors like renal failure, diabetes mellitus, use of broad- spectrum antimicrobial agents etc were observed for biofilm production enhancement. The most common factors associated with Candida infections were the presence of devices, implants and various types of catheters in $49(61.5 \%)$ followed by presence of co-morbid conditions like diabetes mellitus and renal failure $31(38.5 \%)$. The association was observed to be statistically significant with $\mathrm{p}<0.05$. Candida infections were also found to be associated with the increased duration of ICU stay in these patients, with most of the patients staying for more than 2 weeks.

\section{Discussion}

Non albicans Candida spp. once dismissed or ignored as non-pathogenic, commensal has emerged as potential pathogens in last two decades. Among NAC spp. C. tropicalis alone, or in association with other species, is implicated more frequently in human infections.

Candiduria and Candidemia in admitted patients with ongoing indwelling and intravascular catheters is a risk factor for biofilm formation due to suitable niche for adhesion and propagation. Factors such as increased use of antifungal drugs and broad spectrum antibiotics, long- term use of catheters and increase in the number of immunocompromised patients have contributed to 
the emergence of NAC species in significantly high numbers.

This study highlights the prevalence of Candida infection among the hospitalized patients in IPD and ICU, biofilm production and its correlation to rising antifungal resistance. In the present study, the predominance of non-albicans Candida species over C. albicans was a notable feature as more than $60 \%$ of infections were caused by non-albicans Candida, which is in accordance with the published report from various parts of the world. The most common isolate in our study was C. albicans (33\%) followed by C.glabrata $(22 \%)$. The finding in our study was similar with the results reported by Nidhi et al., $2003^{7}$, Jagdish et al., $2013 .^{8}$

Candida spp. have many virulence attributes which assist in invasion of host tissues which include adherence to host tissues, release of extracellular enzymes, production of hyphae to aid in evasion of host immune defences and biofilm production. In comparison to Candida albicans, NAC isolates were shown to produce biofilms in higher proportion especially detected with the help of Microtitre plate. Many studies have reported production of biofilms in range of $20 \%-65 \%$ of NAC isolated using tube and microtitreplate method..$^{9-12}$ In our study biofilm production was seen in $64.9 \%$ of the NAC isolates and $68.7 \%$ of C.albicans isolates. The propensity of biofilm formation in the present study was maximum with Candida tropicalis isolates (71.4\%) followed by Candida albicans $(68.7 \%)$. Among other Non albicans Candida spp like C.krusei, C.dubliniensis and C.guilliermondii, the biofilm production was seen in $20-40 \%$ of isolates.

The tube method showed good correlation with the MTP assay for strongly biofilm forming isolates. However, the chances of false negative were higher in tube method when compared to Microtitre plate method. This can be due to interpersonal observation of biofilm in tube method. The advantage of Microtitre plate method was less time consuming, correct correlation with optical density.

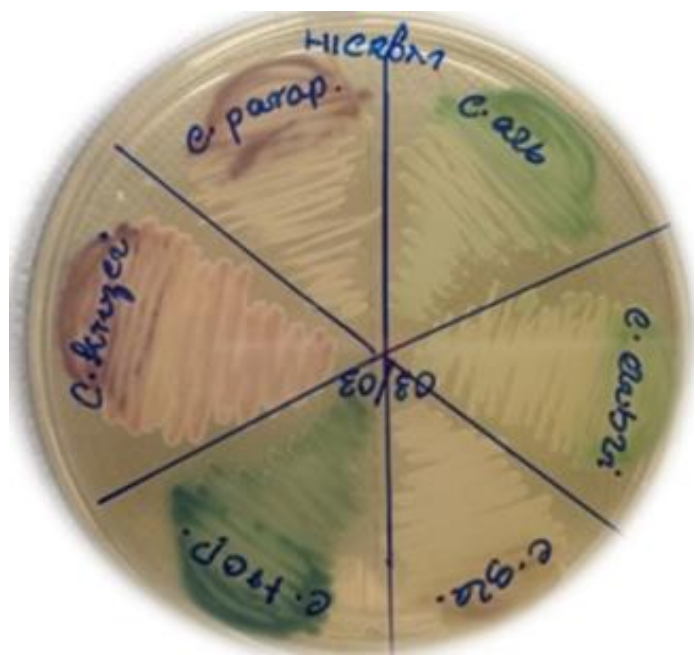

Fig. 1: Gross morphology in CHROMagar

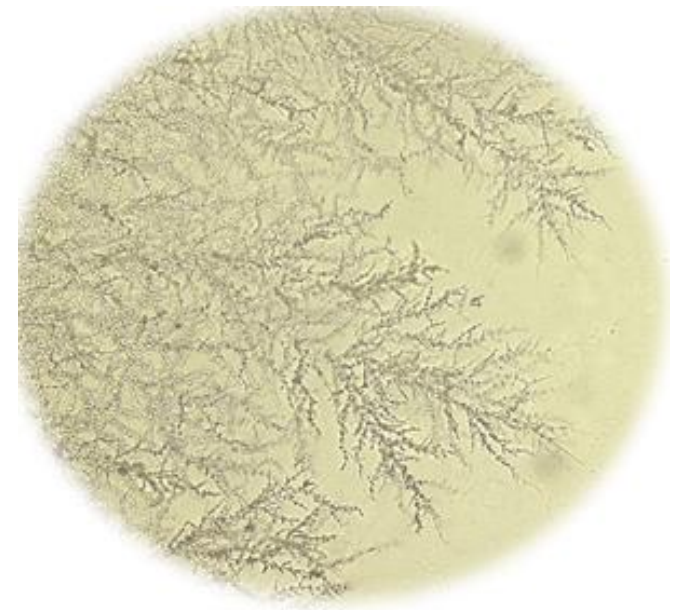

Fig. 2: Microscopic morphology in CMA under 40X

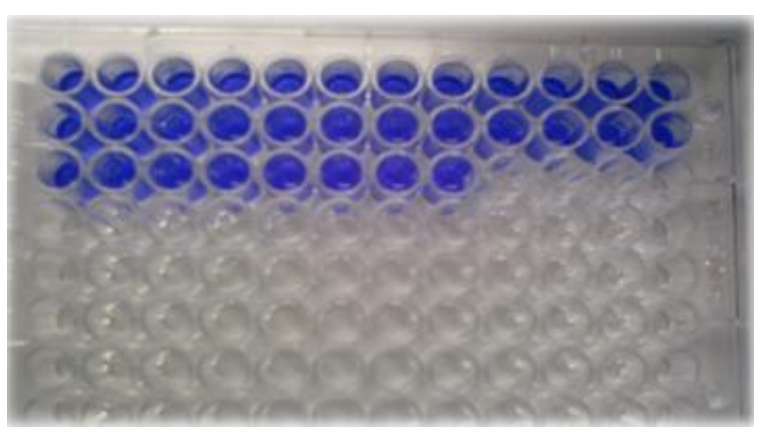

Fig. 3: Biofilm Production using Microtitreplate method 


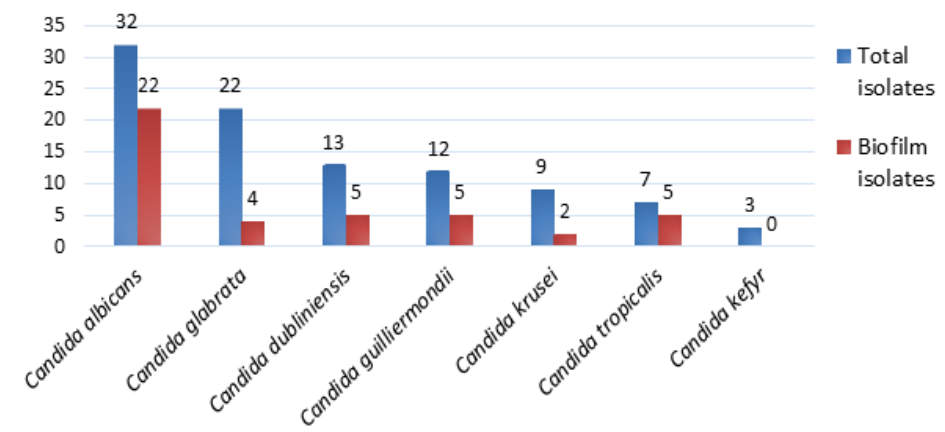

Fig. 4: Biofilm forming Candida species

Table 1: Screening of Candida isolates for the detection of biofilm formation

\begin{tabular}{|l|c|c|}
\hline $\begin{array}{c}\text { Biofilm formation } \\
\text { (mean OD value) }\end{array}$ & \multicolumn{2}{|c|}{ Screening methods } \\
\hline & $\begin{array}{c}\text { Tube } \\
\text { method(\%) }\end{array}$ & $\begin{array}{c}\text { Microtitre plate } \\
\text { method(\%) }\end{array}$ \\
\hline Strong $(>0.320)$ & $18(18.18 \%)$ & $14(14.14 \%)$ \\
\hline Moderate $(0.120-0.320)$ & $9(9.09 \%)$ & $16(16.16 \%)$ \\
\hline Weak $(<0.120)$ & $9(9.09 \%)$ & $13(13.13 \%)$ \\
\hline Non- biofilm producer & $63(63.63 \%)$ & $56(56.56 \%)$ \\
\hline
\end{tabular}

\section{Conclusion}

C. glabrata appears to be the emerging non-C. albicans species at our setup and Non-albicans Candida predominate over $\mathrm{C}$. albicans in nosocomial Candida infections. The overall morbidity and mortality is high especially in critically ill patients and the study highlights the clinical significance of nosocomial Candida infections. Biofilms play a significant role in the perpetuation of these infections primarily with respect to their ability to adhere to various medical devices. Constant monitoring of Candida infections and better interaction between the clinicians and the microbiologists can help in developing strategies and new products with antifungal abilities to control this emerging threat.

Financial support and sponsorship: No support was asked from any funding agency.

Conflicts of Interest: There are no conflicts of interest.

\section{References}

1. Calderone RA, editor. Candida and candidiasis. Washington: ASM Press; 2002.

2. O'Toole GA, Kaplan HB, Kolter R. Biofilm formation as microbial development. Annual Review Microbiol 2000;54:49-79.

3. Donlan RM, Costerton JW. Biofilms: survival mechanisms of clinically relevant microorganisms. Clin Microbiol Rev 2002;15:167-93.

4. Sanglard D, Odds FC. Resistance of Candida species to antifungal agents: molecular mechanisms and clinical consequences. Lancet Infectious Dis 2002;2(2):73-85.

5. National workshop in Medical Mycology. $29^{\text {th }}$ Annual Congress of Indian Association of Medical Microbiologists. MICROCON 2005. Medical Mycology Laboratory Procedures. Sri Ramachandra Medical
College and Research Institute, Porur, Chennai. Oct 2005, 94-115.

6. Clinical and Laboratory Standard Institute. Method for antifungal disc diffusion susceptibility testing for yeasts; approved guidelines. M44-A2. Wayne, PA: CLSI; 2015.

7. Goel N, Ranjan,PK, Aggarwal R, Chaudhary U, Sanjeev N. Emergence of Nonalbicans Candida in Neonatal Septicemia and Antifungal Susceptibility: Experience from a Tertiary Care Center. J Laboratory Physicians 2009;1(2).

8. Chander J, Singla N, Kaur Sidhu S, Gombar S. Epidemiology of Candida blood stream infections: experience of a tertiary care centre in North India. J Infect Dev Ctries 2013;7(9):670-75.

9. Shin JH, Kee SJ, Shin MG, Kim SH, Shin DH, Lee SK, et al. Biofilm production by isolates of Candida species recovered from nonneutropenic patients: comparison of bloodstream isolates with isolates from other sources. $J$ Clin Microbiol 2002;40(4):1244-8.

10. Tumbarello M, Posteraro B, Trecarichi EM, Fiori B, Rossi M, Porta R, et al. Biofilm Production by Candida Species and Inadequate Antifungal Therapy as Predictors of Mortality for Patients with Candidemia. J Clin Microbiol 2007;45(6):1843-50.

11. Deorukhkar SC, Saini S, Mathew S. Virulence Factors Contributing to Pathogenicity of Candida tropicalis and Its Antifungal Susceptibility Profile. Int J Microbiol 2014;2014:1-6.

12. Negri M, Martins M, Henriques M, Svidzinski TIE, Azeredo J, Oliveira R. Examination of Potential Virulence Factors of Candida tropicalis Clinical Isolates From Hospitalized Patients. Mycopathologia 2010;169(3):175-82.

13. Bhatt M, Sarangi G, Paty BP, Mohapatra D, Chayani N, Mahapatra A, Das P, Sahoo D. Biofilm as a virulence marker in Candida species in Nosocomial blood stream infection and its correlation with antifungal resistance. Ind J Med Microbiol 2015;33:S112-4. 